\title{
Aumento no material fibrinoide perivilositário nas placentas de gestações com pré-eclâmpsia
}

Primeira submissão em 16/05/10 Última submissão em 30/08/10 Aceito para publicação em 09/10/10 Publicado em 20/02/11

\author{
Increase in perivillous fibrinoid material in placentas from pregnancies with preeclampsia \\ Diego Agra de Souza'; Antonio Fernando de Sousa Bezerra²; David Campos Wanderley³; Claudia Maria Barbosa Souto ${ }^{3}$
}

unitermos
Pré-eclâmpsia
Placenta
Fibrina

\section{resumo}

Introdução: A pré-eclâmpsia responde por alta morbimortalidade no Brasil e no mundo. A sua etiologia ainda não foi totalmente esclarecida. Entre as alterações placentárias na pré-eclâmpsia citam-se: infartos, aumento de nós sinciciais, hipotrofia vilositária, espessamento da membrana basal trofoblástica e deposição de material fibrinoide. Objetivo: Analisar quantitativamente imagens do material fibrinoide perivilositário presente em placentas de gestações com e sem pré-eclâmpsia. Material e métodos: Foi realizado estudo de caso-controle no Serviço de Anatomia Patológica do Hospital Universitário Professor Alberto Antunes (HUPAA). Realizou-se análise histomorfométrica de 840 imagens provenientes de 14 placentas de gestações com pré-eclâmpsia (casos) e 14 placentas de gestações sem pré-eclâmpsia (controles). Resultados: A média da área do material fibrinoide no grupo pré-eclâmpsia foi de 168,46 pixels, e no grupo sem pré-eclâmpsia, de 89,63 pixels. Foi observada uma quantidade menor da área total dos núcleos na pré-eclâmpsia, 89,51 pixels, do que no grupo controle, 113,34 pixels. Conclusão: Nas placentas de gestações com pré-eclâmpsia a área ocupada pelo material fibrinoide está aumentada em 1,8 vez em comparação com as placentas de gestações normais. As áreas dos núcleos e citoplasmas foram maiores no grupo controle. Não houve diferença estatisticamente significativa quanto à área do estroma. Observou-se também redução do espaço ocupado pelas vilosidades na pré-eclâmpsia, sendo este fato compatível com hipotrofia vilositária.

Introduction: Preeclampsia is responsible for high maternal mortality in Brazil and worldwide. Its etiology has not been fully elucidated. Placental changes in preeclampsia include: infarcts, increase in syncytial knots, villous hypotrophy, thickening of trophoblastic basement membrane and fibrin deposition. Objective: To analyze quantitatively images of perivillous fibrinoid material present in placentas from pregnancies with and without preeclampsia. Material and methods: A case-control study was carried out at the Anatomical Pathological Service of Professor Alberto Antunes University Hospital. It was performed the histomorphometric analysis of 840 images comprising 14 placenta samples from pregnancies with preeclampsia (cases) and 14 placenta samples from pregnancies without preeclampsia (controls). Results: In the preeclampsia group the mean area of fibrinoid material was 168.46 pixels and in the group without preeclampsia it was 89.63 pixels. The nuclei total area was smaller in preeclampsia (89.51 pixels) in comparison with the control group (113.34 pixels). Conclusion: In placentas from preeclampsia pregnancies the fibrinoid material area is larger (1.8x) compared to normal pregnancies. Nuclei and cytoplasm areas were larger in the control group. There was no statistical difference regarding the stromal area. There was also a reduction in villous space in preeclampsia, which is consistent with villous hypotrophy.

1. Estudante do décimo segundo período da graduação em Medicina na Universidade Estadual de Ciências de Saúde de Alagoas (UNCISAL); diretor científico da Liga Acadêmica de Patologia da UNCISAL.

2. Doutor em Anatomia Patológica pelo Hospital Universitário Antônio Pedro da Universidade Federal Fluminense (HUAP/UFF); professor adjunto da Universidade Federal de Alagoas (UFAL) e da UNCISAL.

3. Estudante do oitavo período da graduação em Medicina na UNCISAL; membro da Liga Acadêmica de Patologia da UNCISAL. 


\section{Introdução}

A pré-eclâmpsia é o desenvolvimento de hipertensão e proteinúria em decorrência da gestação ou por influência de gestação recente ${ }^{(9)}$. Ocorre após a 20ª semana de gestação, mas pode surgir antes disso quando existir doença trofoblástica ${ }^{(9)}$. A prevalência de síndromes hipertensivas na gestação é de $6 \%$ a $17 \%$ em nulíparas e de $2 \%$ a $4 \%$ em multíparas ${ }^{(25)}$. Destas, entre $20 \%$ e $50 \%$ progridem para préeclâmpsia ${ }^{(25)}$. A pré-eclâmpsia atinge de $3,64 \%$ a $10 \%$ das mulheres grávidas, sendo a principal causa de mortalidade materna no mundo ${ }^{(14,16)}$. No Brasil e na América Latina também é a principal causa de morte materna, especialmente quando ocorre nas formas mais severas, como eclâmpsia, síndrome HELLP (haemolysis, elevated liver enzyme activity, low platelets), coagulação intravascular disseminada, acidente vascular cerebral (AVC) e insuficiência renal ${ }^{(21)}$.

O aumento da pressão arterial na gravidez pode resultar em maior risco para prematuridade, fetos pequenos para a idade gestacional, infecção neonatal e hipóxia ${ }^{(6)}$. A hipertensão é definida como pressão sistólica $\geq 140 \mathrm{mmHg}$ e pressão diastólica $\geq 90 \mathrm{mmHg}^{(23)}$. O padrão ouro para detectar a proteinúria é a urina de $24 \mathrm{~h}$, que é confirmada com a presença de $300 \mathrm{mg} / 24 \mathrm{~h}$ de proteínas ${ }^{(23)}$. O edema pode ou não estar presente, sendo retirado da tríade clássica sintomática da pré-eclâmpsia ${ }^{(23)}$.

A pré-eclâmpsia é incurável, exceto pela interrupção da gestação(9). A causa primária da pré-eclâmpsia é desconhecida ${ }^{(16)}$, entretanto é reconhecida a importância de aspectos imunológicos, genéticos e de falhas da placentação ${ }^{(16)}$. A invasão trofoblástica deficiente ou ausente, a queda dos níveis de prostaciclina ( $\mathrm{PGI} 2$ ) com aumento do tromboxano A2 e a baixa expressão do antígeno leucocitário humano $\mathrm{G}$ (HLA-G) são integrantes da fisiopatologia(2, 19). O menor influxo de sangue para a placenta resulta em infartos verdadeiros de um ou mais lóbulos placentários ${ }^{(27)}$. O processo de deposição de material fibrinoide entre os vilos ocorre em todas as placentas, sendo sugerido que resulta da cascata de coagulação, da degeneração sinciotrofoblástica ou da secreção trofoblástica ${ }^{(13)}$. No entanto, nas pacientes com pré-eclâmpsia, foi observado que abundante material fibrinoide fica acumulado perivilositariamente ${ }^{(17)}$.

Existem poucos estudos na literatura descrevendo quantitativamente o material fibrinoide em gestantes. A análise estereológica tem sido empregada na medicina com o objetivo de interpretar a morfologia de órgãos e tecidos humanos, permitindo quantificar áreas, volumes e relações espaciais entre os atributos analisados ${ }^{(20)}$. A pergunta da pesquisa é: qual a quantidade de material fibrinoide perivilositário presente em placentas de gestações com e sem pré-eclâmpsia? A hipótese é que a quantidade de material fibrinoide perivilositário depositado nos espaços intervilosos de placentas provenientes de mulheres que desenvolveram pré-eclâmpsia seja maior em comparação com placentas de gestações normais.

\section{Objetivo}

Analisar quantitativamente imagens do material fibrinoide perivilositário presente em placentas de gestações com e sem pré-eclâmpsia.

\section{Material e métodos}

Este estudo foi aprovado pelo comitê de ética em pesquisa da Universidade Federal de Alagoas (UFAL) (Processo no 001672/2007-68). O tipo do estudo é retrospectivo de caso-controle e foi executado no Serviço de Anatomia Patológica do Hospital Universitário Professor Alberto Antunes (HUPAA) em Maceió, no período de agosto de 2007 a dezembro de 2008.

Os critérios de inclusão foram: casos e controles com lâminas coradas em hematoxilina e eosina (HE) apresentando cortes histológicos placentários referentes às gestações no período 1995 a 2000 da maternidade do HUPAA. Os critérios de exclusão foram: casos e controles cujas lâminas de cortes histológicos placentários corados em HE apresentaram qualidade comprometida pela deterioração causada pelo tempo ou pela presença de artefatos, casos e controles nos quais as informações clínicas estavam incompletas e casos com outras doenças superpostas. No total foram selecionadas 28 lâminas, sendo 14 de casos de gestações que cursaram com pré-eclâmpsia e 14 de controles de gestações sem pré-eclâmpsia ou outra doença. Os casos foram definidos como amostras de placentas de gestantes com pré-eclâmpsia fixadas em formol e emblocadas em parafina. Os 14 casos controles foram definidos como amostras de placentas de gestantes sem doenças no decorrer da gestação, todas fixadas em formol e emblocadas em parafina. Destas amostras foram confeccionadas lâminas em HE representando o disco placentário da placa fetal até a placa corial, com a remoção prévia de $3 \mathrm{~cm}$ das margens placentárias. Todos os casos e controles foram oriundos de placentas de gestantes cujos partos foram realizados na maternidade do HUPAA. O diagnóstico foi definido 
pela clínica obstétrica e os achados compatíveis foram descritos por um patologista. Os critérios clínicos utilizados para diagnóstico de pré-eclâmpsia foram: pressão arterial $\geq 140 \times 90 \mathrm{mmHg}$ após a 20 a semana de gravidez e urina de $24 \mathrm{~h}$ com níveis maiores ou iguais a $300 \mathrm{mg}$. Para cada caso foi atribuída uma numeração, sendo a análise de imagens executada cegamente.

Para a obtenção das imagens foi utilizada uma câmera digital Moticam 1000, um microscópio Leica DMLS e um computador Acer Aspire 3660. Neste estudo foi realizada uma análise morfométrica planimétrica ${ }^{(3)}$. A máquina foi acoplada à ocular do microscópio e interligada com o computador. As lâminas e as objetivas do microscópio foram limpas antes da captação das imagens, evitando a inclusão de manchas na imagem. As imagens foram armazenadas no banco de dados do computador no formato tif. Todas as imagens foram obtidas do terço intermediário, tendo sido descartados os terços que abrangem, respectivamente, as placas fetal e materna. Durante o processo de obtenção foi utilizado o mesmo microscópio com igual ajuste de iluminação e filtro DLF. O aumento escolhido foi de 400x. Para cada lâmina de placenta foram obtidas 30 imagens. Esta técnica foi baseada em outras pesquisas descritas na literatura ${ }^{(6)}$. Dessa forma, obtiveram-se 840 imagens de 14 casos de pré-eclâmpsia e 14 casos sem pré-eclâmpsia.
O processamento de imagens foi realizado utilizando o programa Animed ${ }^{(4)}$. As imagens englobaram os vilos maduros intermediários e terminais. Foram definidos cinco componentes histológicos: material fibrinoide, núcleos, estroma, citoplasma e fundo ${ }^{(12)}$. Nos componentes citoplasma e núcleos são incluídos os núcleos e citoplasmas de todas as células presentes na estrutura dos vilos terminais $\mathrm{e}$ dos vilos maduros intermediários. Predominantemente são encontrados entre as células o sinciotrofoblasto, as células do estroma (fibroblastos, células reticulares), seguidos das células endoteliais e, mais raramente, dos macrófagos e das células citotrofoblásticas ${ }^{(12)}$. O estroma corresponde ao tecido conectivo localizado na região mais central do vilo, abaixo das células do sinciotrofoblasto e entre os capilares, onde estão imersos os fibroblastos e as células reticulares. O fundo representou o espaço existente entre as estruturas vilositárias.

Uma imagem digital é formada por um conjunto de pixels. O pixel corresponde à menor área retangular da imagem ${ }^{(3)}$. Após uma seleção prévia de alguns pixels correspondente a cada componente observado na estrutura do vilo (núcleos, material fibrinoide, estroma, citoplasma e fundo), o software segmentou a imagem segundo os pixels selecionados, informando a quantidade percentual da área ocupada por cada componente analisado em cada imagem (Figura). Em seguida foi realizada a conversão do

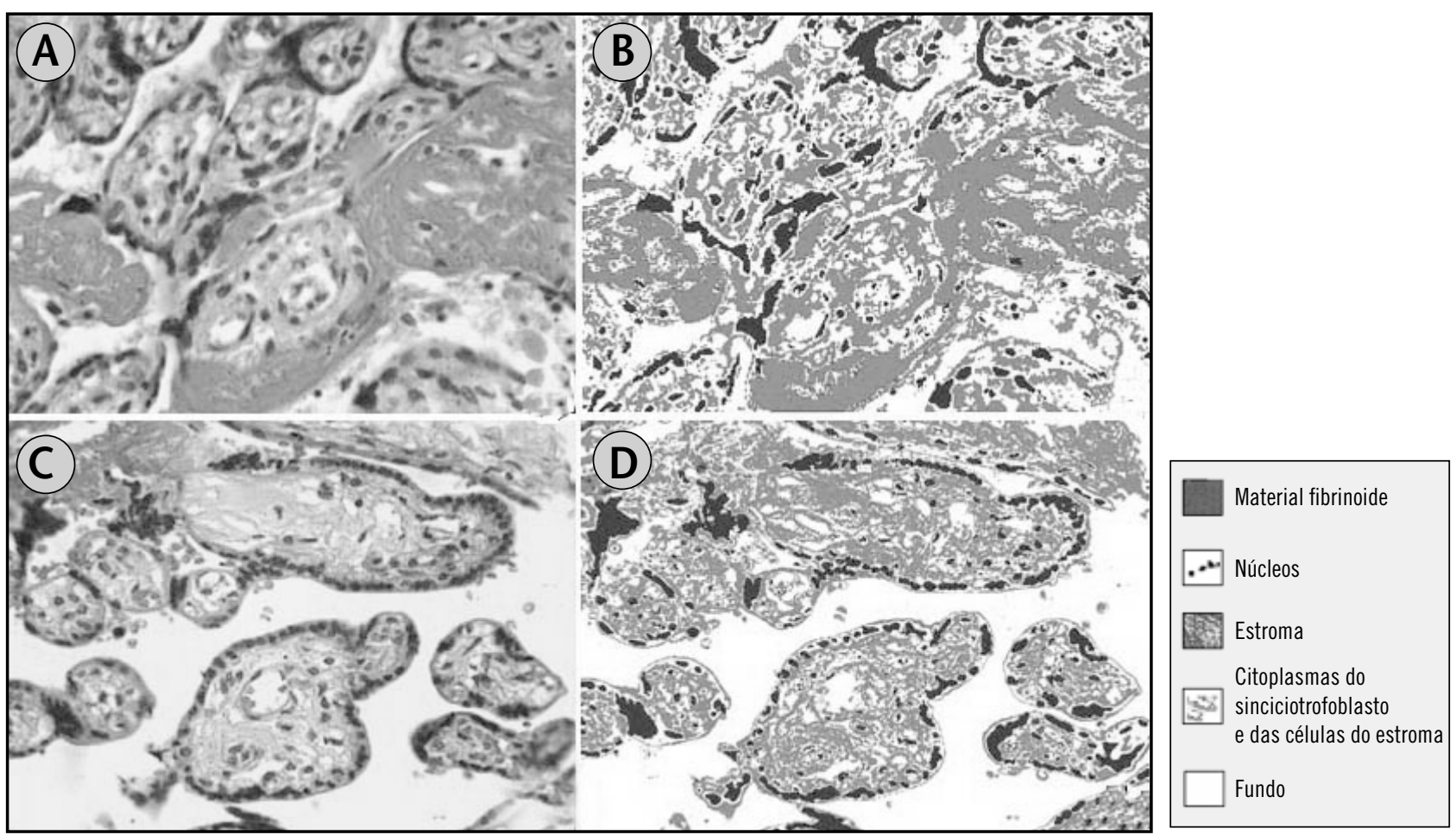

Figura 1 - (A) Imagem de placenta de gestação com pré-eclâmpsia (HE, 400 x); (C) imagem de placenta de gestação sem pré-eclâmpsia (HE, 400 x); (B e D) imagens segmentadas (ao lado de suas originais) para quantificação do material fibrinoide; núcleos; estroma; citoplasmas do sinciciotrofoblasto e das células estromais e do fundo

Nota: ver o quadro de amostragem ao lado das imagens como substituição das cores originais na indicação das estruturas segmentadas. 
valor percentual para o valor real de pixels. Os dados da área foram armazenados no programa Epi Info versão 617. A estatística foi calculada usando-se o programa XLSTAT 200918. Foi realizado pré-teste com as colorações azul de toluidina, tricrômio de Masson, ácido periódico Schiff (PAS) e HE. Optou-se pela coloração HE com base nos resultados deste pré-teste e em dados da literatura ${ }^{(6)}$. A variável primária considerada foi a área ocupada pelo material fibrinoide. As variáveis secundárias incluem as áreas ocupadas por núcleos, estroma, citoplasma, fundo e a correlação das áreas dos atributos entre si.

$\mathrm{Na}$ análise estatística foram calculados: média, mediana, intervalo de confiança, desvio padrão, coeficiente de variação e distância interquartílica. $A$ análise estatística foi baseada no valor real dos pixels. A amostra foi considerada de distribuição não normal quando o coeficiente de variação esteve acima de $20 \%$. Para comparar as diferenças entre os componentes histológicos utilizou-se o teste MannWithney. Considerou-se o $\alpha=0,05$ e os resultados foram considerados significativos quando $p<0,05$.

\section{Resultados}

No período de 1995 a 2000 foram encontrados registros de 4.343 exames histopatológicos de placenta. Cento e vinte e dois foram de casos diagnosticados como préeclâmpsia, 68 casos foram excluídos por haver outras doenças superpostas, restando 54 casos. Entre estes, 29 foram excluídos porque as lâminas ou os prontuários não foram localizados. Dos 25 pré-selecionados, 11 foram descartados porque apresentaram lâminas deterioradas ou com artefatos. Todos os 14 casos selecionados foram de pré-eclâmpsia classificada clinicamente como grave. Foram selecionados 14 controles aleatoriamente de um total de 142 casos sem pré-eclâmpsia ou outras morbidades, usando-se o gerador de números aleatórios do Microsoft Oficce Excel ${ }^{(22)}$. Foram obtidas 30 imagens de cada caso e controle.

Os dados clínicos podem ser observados na Tabela 1. A média da área do material fibrinoide no grupo préeclâmpsia foi de 168,46 pixels e no grupo controle, de 89,63 pixels. Essa diferença representa uma quantidade 1,8 vez maior em relação ao grupo controle (Tabela 2 ) $(p<0,0001)$. Foi observada uma quantidade menor da área total dos núcleos na pré-eclâmpsia, 89,51 pixels, do que no grupo controle, 113,14 pixels (Tabela 2$)(p<0,0001)$, e a área do citoplasma e do fundo foi maior no grupo controle (Tabela 2) $(p<0,0001)$. A diferença encontrada entra as áreas ocupadas pelo estroma não foi estatisticamente significativa $(p=0,216)$. A área ocupada pelos vilos, que corresponde às áreas somadas do citoplasma, dos núcleos e do estroma, foi maior no grupo controle $(p<0,0001)$. Foi encontrada uma correlação negativa $(-0,40976)$ fraca entre a área ocupada pelos vilos e o material fibrinoide no grupo pré-eclâmpsia. Em todas as variáveis analisadas foi observada distribuição não normal, com coeficiente de variação superior a $20 \%$. O teste de Mann-Whitney foi aplicado para comparar as diferenças dos dois grupos não pareados com distribuição não normal.

\section{Discussão}

Entre as alterações morfológicas placentárias das gestações que cursam com pré-eclâmpsia estão: infartos, aumento de nós sinciciais, aterose das artérias espiraladas, hipotrofia vilositária, espessamento da membrana basal trofoblástica e deposição de material fibrinoide $(17,24,26,27)$.

O material fibrinoide placentário é uma substância hialina, homogênea que se cora em vermelho pela eosina ${ }^{(26)}$. É comum utilizar os termos fibrina e fibrinoide como sinônimos ${ }^{(26)}$. No entanto, outros autores preferem

\section{Tabela 1 Dados clínicos}

\begin{tabular}{lcccc}
\hline & Pré-ECLÂMPSIA* & IC 95\% & CONTROLE* $^{*}$ & IC 95\% \\
Idade gestacional (semanas) & 36,08 & $34,38-37,78$ & 38,25 & $37,39-39,11$ \\
Idade materna (anos) & 24,21 & $21,66-26,76$ & 25,21 & $22,28-28,14$ \\
Peso do RN (gramas) & 2.931 & $2.673-3.157$ & 3.012 & $2.847-3.177$ \\
Apgar 1 & 7,14 & $5,65-8,63$ & 8,35 & $8,09-8,61$ \\
Apgar 5 & 8,28 & $6,98-9,58$ & 9,28 & $8,96-9,6$ \\
\hline
\end{tabular}

*Valores expressos em média.

IC: intervalo de confiança; $R N$ : recém-nascido. 
Tabela 2 Variáveis primárias e secundárias

\begin{tabular}{lccccccc}
\hline Pré-eclâmpsia & Média & Mediana & Amplitude & DI & IC 95\% & DP & CV (\%) \\
Material fibrinoide & 168,46 & 150,62 & 487,58 & 151,59 & $158,56-178,36$ & 103,15 & 61,23 \\
Núcleos & 89,51 & 149,9 & 520,87 & 122,71 & $83,48-95,54$ & 62,44 & 69,75 \\
Fundo & 273,20 & 261,04 & 677,29 & 150,69 & $261,76-284,64$ & 118,82 & 43,91 \\
Estroma & 166,8 & 166,19 & 385,26 & 99,07 & $159,88-173,38$ & 71,87 & 43,08 \\
Citoplasma & 88,64 & 64,61 & 337,46 & 92,93 & $81,98-95,3$ & 69,24 & 78,11 \\
Cit. + núc. + est. & 345,56 & 305,37 & 1243,09 & 275,61 & $326,23-364,89$ & 200,49 & 58,01 \\
Controle & Média & Mediana & Amplitude & DI & $1 C 95 \%$ & DP & CV (\%) \\
Material fibrinoide & 89,63 & 85,73 & 257,22 & 70,25 & $84,39-94,87$ & 54,66 & 60 \\
Núcleos & 113,34 & 114,78 & 249,68 & 82,79 & $108,31-118,37$ & 52,47 & 45,71 \\
Fundo & 296,19 & 302,06 & 497,19 & 144,93 & $286,33-306,05$ & 102,87 & 34,73 \\
Estroma & 174,94 & 164,03 & 413,01 & 111,55 & $168,06-181,82$ & 71,76 & 41,01 \\
Citoplasma & 110,85 & 109,46 & 275,97 & 61,78 & $106,51-115,19$ & 45,24 & 40,81 \\
Cit. + núc. + est. & 401,37 & 368,85 & 881,58 & 256,12 & $385,21-417,53$ & 168,27 & 41,92 \\
\hline
\end{tabular}

DI: distância interquartílica; IC: intervalo de confiança; DP: desvio padrão; CV: coeficiente de variação; cit. + núc. + est.: citoplasma + núcleos + estroma.

o termo fibrinoide por ser mais abrangente e restringem o termo fibrina aos precipitados de fibrinogênio no sangue e fluidos teciduais ${ }^{(13)}$. A formação fibrinoide não está restrita a processos patológicos e ocorre em placentas normais ${ }^{(13)}$. $\mathrm{Na}$ placenta, está presente em diferentes locais: placa coriônica, perivilositariamente, intravilositariamente e na placa basal, recebendo diferentes epônimos de acordo com a localização (estria de Langhans, estria de Rohr, estria de Nitabuch) ${ }^{(18)}$. A despeito da localização anatômica, estudos com imuno-histoquímica foram realizados objetivando estudar a composição do material fibrinoide em suas variadas localizações. Tais pesquisas permitiram dividir o material fibrinoide em dois grupos: tipo fibrina e tipo matriz ${ }^{(13,15,18)}$.

O material fibrinoide tipo fibrina é considerado um produto da coagulação sanguínea materna, sendo encontrado depositado perivilositariamente, sobre a placa coriônica, nas camadas superficiais e mais profundas da placa basal ${ }^{(13,15,18)}$. O estudo microscópico revela a ausência de células trofoblásticas em seu interior, porém é possível encontrar material degenerativo de hemácias, plaquetas e células do sinciotrofoblasto ${ }^{(13,18)}$. À microscopia eletrônica é observada a presença de fibras com periodicidade de $20 \mathrm{~nm}$, revelando um padrão característico da fibrina ${ }^{(13,18)}$. A análise da imunohistoquímica revela reação intensa do anticorpo antifibrina com este tipo de material fibrinoide ${ }^{(18)}$. Imunoglobulinas
(lgG, $\lg A, \lg M)$, fibrinogênio, albumina e complemento $C$ são outros componentes descritos como constituintes do material fibrinoide tipo fibrina ${ }^{(18)}$. Quanto ao processo que desencadeia a deposição do material fibrinoide tipo fibrina, são levantadas duas hipóteses ${ }^{(18)}$ : a primeira descreve que a deposição tem início após a perda do sinciotrofoblasto, e a segunda, que a coagulação provoca degeneração sinciotrofoblástica ${ }^{(18)}$. Essas duas hipóteses foram suscitadas levando em consideração o fato de que o material fibrinoide tipo fibrina cobre áreas desnudas do sinciotrofoblasto ${ }^{(18)}$. Ambas são defendidas, sendo a formação do material fibrinoide induzida pelo duplo mecanismo ${ }^{(18)}$. A provável exposição de moléculas da matriz extracelular interage com as plaquetas, dando origem ao material fibrinoide ${ }^{(18)}$. Entre as funcionalidades postuladas para o material fibrinoide tipo fibrina estão: conferir a estabilidade a placenta, participar de mecanismos de reparação do vilo e regular a circulação entre os vilos ${ }^{(18)}$.

O material fibrinoide tipo matriz é um produto da secreção das células trofoblásticas, razão pela qual estas são facilmente encontradas no seu interior ${ }^{(13,18)}$. O material fibrinoide com constituição da matriz não está em contato direto com o sangue materno e é sempre separado por uma camada de material fibrinoide tipo fibrina ou por outros tecidos $^{(13,18)}$. Está localizado em: placa coriônica, centro das ilhas de células, mais profundamente nas colunas de 
células trofoblásticas, placa basal e camadas mais profundas do material fibrinoide perivilositário(13, 18). Entre os componentes do material fibrinoide tipo matriz estão: colágeno IV, laminina, fibronectinas, sulfato de heparan, vitronectina, fibronectina oncofetal e fibrilina ${ }^{(13,15,18)}$. O mascaramento de antígenos fetais e a regulação da adesividade placentária à parede uterina são algumas das funções propostas para o material fibrinoide tipo matriz ${ }^{(18)}$. A proporção dos dois subtipos de materiais fibrinoides varia conforme o local da placenta. A subdivisão entre material fibrinoide derivado da coagulação e da secreção de células trofoblásticas independe da localização anatômica ${ }^{(13)}$.

O material fibrinoide está aumentado perivilositariamente na hipertensão gestacional, na hipertensão crônica e na pré-eclâmpsia ${ }^{(6,17,22)}$. Neste estudo foi demonstrado um aumento de aproximadamente 1,8 vez no material fibrinoide, predominantemente, perivilositário no grupo pré-eclâmpsia em relação ao grupo controle $(p<0,0001)$. Na literatura, Corrêa et al.(6) relatam aumento mais discreto, ocupando a fibrina a área de $4 \%$ no grupo controle e $4,4 \%$ da área total nas gestações prematuras com pré-eclâmpsia ${ }^{(6)}$. Kanfer et al. ${ }^{(17)}$ descreveram que a porcentagem de vilos com depósitos fibrinoides perivilositários foi maior em placentas acometidas pela pré-eclâmpsia: $4,8 \%$ contra $1,5 \%$ no grupo controle; um aumento de 3,2 vezes ${ }^{(17)}$. Essas variações entre os estudos se devem, provavelmente, à metodologia de seleção empregada. Neste estudo foram incluídas as imagens captadas de áreas com uma quantidade representativa de material fibrinoide perivilositário no terço médio da placenta. Para diminuir as chances de um aumento de viés, o mesmo procedimento foi repetido nos casos e controles.

Na pré-eclâmpsia também se nota uma diminuição da área das vilosidades placentárias ${ }^{(5)}$. Corrêa et al. ${ }^{\left({ }^{(6)}\right.}$ consideram que a diminuição dos vilos está associada ao aumento do material fibrinoide. Porém dois estudos de análise de imagens realizados com placentas de pré-eclâmpsia e com placentas de casos com restrição do crescimento intrauterino concluíram que não foram encontradas alterações no grupo da pré-eclâmpsia quando em comparação com o grupo controle ${ }^{(7,10)}$. Neste estudo, quando somamos a área dos núcleos, estroma e citoplasma, é obtida, de modo indireto, a área correspondente ao espaço ocupado pelas vilosidades. O grupo pré-eclâmpsia apresenta uma área de vilos menor que o grupo controle $(p<0,0001)$. Porém, uma correlação negativa fraca $(-0,4)$ foi encontrada entre a área dos vilos placentários e a área ocupada pelo material fibrinoide no grupo pré-eclâmpsia, indicando que essa diminuição não se deve exclusivamente ao aumento do material fibrinoide. Esse achado sugere uma hipotrofia vilositária nas placentas do grupo pré-eclâmpsia. Ao analisar-se isoladamente a área ocupada pelos núcleos, observa-se uma diminuição significativa no grupo pré-eclâmpsia. A redução da área ocupada pelos núcleos no grupo pré-eclâmpsia é indicativa de que há degeneração celular.

\section{Conclusão}

Nas placentas de gestações com pré-eclâmpsia a área ocupada pelo material fibrinoide está aumentada em 1,8 vez em comparação com as placentas de gestações normais. As áreas dos núcleos e citoplasmas foram maiores no grupo controle. Não houve diferença estatisticamente significativa quanto à área do estroma. Observou-se também uma redução do espaço ocupado pelas vilosidades na pré-eclâmpsia, sendo este fato compatível com hipotrofia vilositária.

\section{Referências}

1. ADDINSOFT. XLSTAT is the leading data analysis and statistical solution for Microsoft Excel. New York. 2009. Disponível em: <http://www.xlstat.com>. Acesso em: 10 out. 2009.

2. ALVES, C. et al. O papel do complexo principal de histocompatibilidade na fisiologia da gravidez e na patogênese de complicações obstétricas. Rev Bras Saúde Matern Infant, v. 4, p. 357-63, 2007.

3. ANDREA, C. E.; BLEGGI-TORRES, L. F.; ALVES, M. T. S. Análise da morfometria nuclear: descrição da metodologia e o papel dos softwares de edição de imagem. J Bras Patol Med Lab, v. 44, n. 1, p. 51-7, 2008.
4. BEZERRA, A. F. S. et al. Animed. Recife. 2004. 1 CD-ROM.

5. BOYD, P. A.; SCOTT, A. Quantitative structural studies on human placentas associated with pre-eclampsia, essential hypertension and intrauterine growth retardation. Br J Obstet Gynaecol, v. 92, p. 714-21, 1985.

6. CORREAA, R. R. M. et al. Placental morphometrical and histopathology changes in the diferent clinical presentations of Hypertensive Syndromes in Pregnancy. Arch Gynecol Obstet, v. 277, p. 201-6, 2008.

7. DAAYANA, S.; BAKERER, P.; CROCKER, I. an image analysis technique for the investigation of variations in placental morphology in pregnancies complicated 
by preeclampsia with and without interauterine growth restriction. Journal of the Society for Gynecologic Investigation, v. 11, n. 8, p. 545-52, 2004.

8. DEAN, A. G. et al. Epi Info, Version 6: a word processing, database, and statistics program for public health on IBM-compatible microcomputers. Centers for Disease Control and Prevention, Atlanta, Georgia, USA, 1996.

9. DUSSE, L. M. S.; VIEIRA, L. M.; CARVALHO, M. G. Avaliação do dímero $D(D-D i)$ na doença hipertensiva específica da gravidez (DHEG). J Bras Patol Med Lab, v. 39, n. 3, p. 199-202, 2003.

10. EGBOR, M. et al. Pre-eclampsia and fetal growth restriction: how morphometrically different is the placenta? Placenta, v. 27, p. 727-34, 2006.

11. FOX, H.; SEBIRE, N. The development and structure of the placenta. Pathology of the Placenta. 3. ed. China: Elsevier, 2007. p. 40.

12. FOX, H.; SEBIRE, N. The development and structure of the placenta. Pathology of the Placenta. 3. ed. China: Elsevier, 2007. p. 28-33.

13. FRANK, H-G. et al. Imunohistochemistry of two different types of placental fibrinoid. Acta Anat, v. 150, p. 55-68, 1994.

14. GONÇALVES, R.; FERNANDES, R. A. Q.; SOBRAL, D. $H$. Prevalência da doença hipertensiva específica da gestação em hospital público de São Paulo. Rev Bras Enferm, v. 58, n. 1, p. 61-4, 2005.

15. HUPPERTZ, B. The anatomy of the normal placenta. J Clin Pathol, v. 61, p. 1296-302, 2008.

16. KAHHALE, S.; ZUGAIB, M. Pré-eclâmpsia. In: BENZECRY, R.; OLIVEIRA, H.C.; LEMGRUBER, I. (eds). Tratado de obstetrícia FEBRASGO. Rio de Janeiro: Revinter, 2000. p. 524-9.
17. KANFER, A. et al. Increased placental antifibrinolytic potential and fibrin deposits in pregnancy-induced hypertension and preeclampsia. Lab Invest, v. 74, n. 1, p. 253-8, 1996.

18. KAUFFMANN, P.; HUPPERTZ, B.; FRANK, H-G. The fibrinoids of the human placenta: origin, composition and functional relevance. Ann Anat, v. 178, p. 485-501, 1996.

19. MATHAI, M. Pre-eclampsia. Ceylon Med J, v. 41, n. 1, p. 7-9, 1996.

20. MAYHEW, T. M. A stereological perspective on placental morphology in normal and complicated pregnancies. J Anat, v. 215, n. 1, p. 77-90, 2008.

21. MELO, B. C. P. et al. Perfil epidemiológico e evolução clínica pós-parto na pré-eclâmpsia grave. Rev Assoc Med Bras, v. 55, n. 2, p. 175-80, 2009.

22. MICROSOFT CORPORATION. Microsoft Office Excel 2007. Redmond, Wa. 2007. 1 CD-ROM.

23. MONTENEGRO, C. B. A.; FILHO, J. R. Toxemia gravídica: pré-eclâmpsia/eclâmpsia. Rezende Obstetrícia Fundamental. 11. ed. Rio de Janeiro: Guanabara Koogan, 2008. p. 206-17.

24. NISHIMURA, Y. et al. Pathological studies on the placenta from pre-term and term maternal toxicosis. Kobe J Med Sci, v. 40, n. 1, p. 13-24, 1994.

25. OLIVEIRA, C. A. et al. Síndromes hipertensivas da gestação e repercussões perinatais. Rev Bras Saúde Matern Infant, v. 6, n. 1, p. 93-8, 2006.

26. SALA, M. A. et al. Ultrastructure of the villous fibrinoid in the human full-term placenta. Rev Chil Anat Temuco, v. 15, n. 1, 1997.

27. SOMA, H. et al. Morphologic changes in the hypertensive placenta. Contrib Gynecol Obstet, v. 58, p. 58-75, 1982. 